Volume 8. No. 2, February 2020

International Journal of Emerging Trends in Engineering Research

Available Online at http://www.warse.org/IJETER/static/pdf/file/ijeter03822020.pdf

httbs://doi.org/10.30534/iieter/2020/03822020

\title{
A Mobile Malaria Fever Clinical Diagnosis System Based on Non-Nested Generalized Exemplar (NNGE)
}

\author{
Oguntimilehin A. \\ Department of Computer Science, Afe Babalola University, Ado-Ekiti, Nigeria, ebenabiodun2@yahoo.com
}

\begin{abstract}
The world today is a world of internet technology which has been deployed in all fields of endeavours to change the way humans do their daily activities and turn things around positively. The whole world is full of smart phones that house various mobile applications. A mobile application for malaria diagnosis was developed in this work to provide a wider coverage and accessibility. A data mining technique Non-Nested Generalized Exemplar (NNGE) was used on malaria labelled dataset collected from a reputable hospital in Ado-Ekiti, Ekiti State, Nigeria to generate a classification model for malaria diagnosis. The model was tested on both the training and testing sets, attaining $100 \%$ and $98.04 \%$ detection rates respectively. The rules generated by NNGE were used as the inference engine of the user friendly mobile diagnosis application. The mobile app was developed using Java, HTML and PhP as front end, MySQL as the backend and Apache as the webserver.
\end{abstract}

Key words: Data Mining, Diagnosis, Machine Learning, Malaria Fever, Mobile Application, NNGE.

\section{INTRODUCTION}

Research worldwide is concentrating on the development of new applications in the medical field and particularly in diagnosis [1]. The world today is one with growing access to intelligent systems. Artificial Intelligence (AI) has been successfully applied in all areas of life in our societies such as education, healthcare, finance and commerce among others [2]. Currently, AI has been meaningfully exploited in medical applications and research efforts have been focused on medical expert systems as a balancing solution to conventional technique for finding solutions to medical problems. The advent of Information Technology (IT) has created exceptional opportunities in health care delivery system and request for intelligent and knowledge-based systems has improved as modern medical practices become more knowledge-demanding [3]. Computer is better than humans when it comes to remembering things and such asset is very useful for computer-aided systems [4]. In AI, expert systems are created and they use the knowledge domain stored in the computer to provide meaningful solutions to problems [5].
Telemedicine is a method of taking health information across distances. Telecommunication devices like internet and telephone are being used to actualise this. Though telemedicine has been considered as one solution by which necessary health care services can be provided to everybody at a reasonable cost, the practice should be treated with caution to do not jeopardise the health sector [6]. Delivery health care services through telemedicine can be traced back to the invention of telephone and television in the 19th century. Technologically advanced countries have exploited Information and Communication Technology (ICT) potentials in making health delivery available at homes [7].

Mobile wireless technology is any technology that uses radio frequency spectrum in any band to facilitate transmission of text, data, voice, and video (multimedia) services to mobile devices at anytime and anywhere. With the introduction of mobile network data services such as General Packets Radio Services (GPRS), Enhanced Data rates for GSM Evolution (EDGE) and Third Generation (3G) in particular, ground-breaking applications and services are gradually becoming popular. A mobile device is no longer considered being luxury item rather it is considered being necessity [8] and today we are talking of the fifth Generation (5G). New fibre and wireless infrastructure, as well as the rapid growth of computer processing power, provide an unprecedented opportunity to scale up health worker training and improve its quality. It optimizes health services delivery and strengthens health systems [9]. In most tropical countries, most of which are developing countries, medical personnel and facilities are not adequate for effective tackling of tropical diseases. Intelligent systems have become vital in the growth and survival of healthcare sector [3].

The increased in demand for high-quality medical services coupled with the explosive growth of medical knowledge led to the suggestion that computer programs should be used in assisting physicians and other healthcare providers in discharging their clinical roles such as diagnosis, therapy and treatment [10]. Malaria is a mosquito borne infectious diseases caused by a eukaryotic protist of the genus plasmodium. It is wide spread in tropical and subtropical regions, including parts of the American, Asia and African [11].

Data mining is the process of discovering interesting patterns from massive amounts of data. As a knowledge discovery process, it typically involves data cleaning, data integration, data selection, data transformation, pattern discovery, pattern 
evaluation, and knowledge presentation. A pattern is fascinating if it is effective on test data with some degree of certainty, novel, potentially useful and easily understood by humans. Interesting patterns represent knowledge. Measures of pattern interestingness, either objective or subjective, can be used to guide the discovery process. The key scopes are data, knowledge, technologies, and applications. Data mining can be conducted on any kind of data as long as the data are meaningful for a target application [12]. Classification is a common term in data mining and it is a practice in which test sample is given a class based on the training received by a model [13]. Machine learning usually refers to the changes that perform tasks associated with artificial intelligence (AI). Such tasks involve recognition, diagnosis, planning, robot control, prediction and so on [14].

\section{REVIEW OF RELATED LITERATURE}

Some earlier systems in the area of the subject matter are presented in this section; they also serve as part of the motivations for this work.

The work of Adetunmbi et al. in [15] presented a Web-Based Medical Assistant System for Malaria Diagnosis and Therapy. Motivations for this work include- most of the available systems on malaria diagnosis do not provide treatment while some focused on treatment without diagnosis, $50 \%$ of people globally are at the risk of malaria and deaths associated with malaria are at increasing rate. Rough Set was used to generate explainable diagnosis rules in this work. The system was implemented as a web-based application. Fuzzy Technique was employed for the management of malaria in [3]. The authors highlighted malaria to be a great threat to some communities in the world and the medical practice has become very complex which requires much attention. Fuzzy techniques were used on the data collected and fuzzy expert system was developed for the management of malaria.

A system for identifying different species of malaria parasites was developed in [16]. The need for timely diagnosis so as to reduce deaths in particular necessitated this research. Two expert systems were developed to achieve the diagnosis of malaria. The medical expert system was created by a rule based on decision support tool (CLIPS). Certain assumptions were made to formalise the knowledge in both the rule based and Bayesian systems. Linear Programming was used to diagnose malaria in [17]. The research motivations include malaria affects between 300 million to 500 million people and kills more than a million people yearly. Linear Programming model was formulated from given malaria dataset in this work.

Decision Support System for Malaria and Dengue Disease Diagnosis (DSSMD) was presented in [18]. The researchers embarked on this project because malaria and dengue fever are the most common cause of deaths in India and other tropical countries of the world. MATLAB was used to develop the system using Fuzzy Logic Tool Box. Graphical User Interface (GUI) which displays the symptoms, knowledge base which involves fuzzification and inference engine which involve defuzzification are the three modules of the system developed. Having a good diagnostic system that is mobile application based may give a better accessibility. A Knowledge Based Expert System for Symptomatic Automated Healthcare was developed in [19]. The expert system developed has three client modules. The modules are user interface, knowledge base and inference engine. Patients interact with the system by supplying symptoms after which diagnosis is done and treatment is provided. The basis on which the diagnosis rules were generated was not mentioned, detection rate was lacking among others.

\section{METHODOLOGY}

\subsection{Collection and Description of Dataset}

Data on symptomatic diagnosis of malaria fever was collected from Adetoyin hospital in Ado-Ekiti, Nigeria. The medical experts classified each record using the available symptoms. There are five different classes (Very High, High, Moderate, Low and Very Low) and a total of nineteen conditional attributes (symptoms) under consideration. The conditional attributes (symptoms) are: Anorexia (ANR), Headache (HEC), Catarrh (CAH), Insomnia (ISN), Yellow Urine (YEU), Vomiting (VOM), Joint Pain (JOP), Dizziness (DSN), Ill-looking (ILL), Convulsion (COV), Body Temperature (BOT) and Diarrhea (DIA), Weakness (WKN), Abdominal Pain (ABP), Cough (COH), Body Pain (BOP), Fever (FVR), Rigour (RGR), Cold (COD). Each instance of the data set corresponds to a medical record of a patient. Each conditional attribute takes a value from High, Low or None depending on the patient's feelings. One thousand, two hundred and twenty five instances served as training set, while four hundred and eight instances served as testing set.

\subsection{Description of the Data mining Technique Used: Non-Nested Generalized Exemplar (NNGE)}

This technique is Nearest-neighbour-like algorithm using non-nested generalized exemplars, which are hyper rectangles that can be viewed as if-then rules [20, 21]. NNGE classifies new examples by determining the nearest neighbour in the exemplar/hyper rectangle database using a Euclidean distance function. This function is modified slightly to enable it to compute the distance from hyper rectangles. The function (identical to that used by Nested Generalized Exemplar (NGE)) is:

$\left.D_{E H}=W_{H} \sqrt{\sum_{i=1}^{m}(W i} \frac{E_{i}-H_{i}}{\max _{i}-\min _{i}}\right)^{2}$

where $E i$ is the $i$ th feature value in the example, $H i$ is the $i$ th feature value in the exemplar, and $W H$ and $W i$ are exemplar 
Oguntimilehin A., International Journal of Emerging Trends in Engineering Research, 8(2), February 2020, 259 - 264

and feature weights. The feature difference $E i-H i$ for ungeneralised exemplars is the difference between the feature value of the example and that of the exemplar, while for hyperrectangles it is defined in Equation 2.

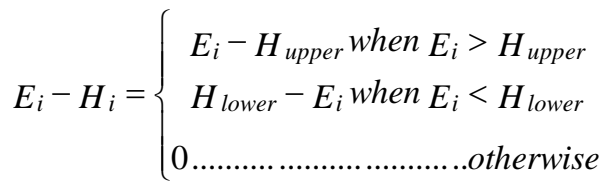

where Hupper and Hlower are the boundaries of the hyperrectangle for this feature. For symbolic attributes, the distance is trivialised to

$E i-H i()=0$ if $E i$ is in the exemplar/hyperrectangle

$E i-H i()=1$ if $E i$ is not in the exemplar/hyperrectangle

Having computed the distance between the new example and all exemplars and hyperrectangles, NNGE chooses the class of the closest one. In the event of a tie, it chooses the class with the most exemplars at the minimum distance. NNGE treats missing attributes by ignoring them; if the attribute is missing for either the example or the exemplar against which it is being compared, it does not contribute to the distance function. The final distance is divided by the number of non-missing attributes so that exemplars with all attribute values present are not penalized [22].

NNGE is one of the machine learning techniques implemented in the Waikato Environment for Knowledge Analysis data mining tool. For further details, consult [23]

\section{EXPERIMENTAL SETUP AND RESULTS}

NNGE used the training set to build a classification model; the model was tested on the training set and testing set. The results obtained are displayed in the Table 1 and Table 2.

Table1: Confusion Matrix of the training set

\begin{tabular}{|l|l|l|l|l|l|}
\hline $\begin{array}{l}\text { Predicted as } \\
\text { Actual }\end{array}$ & V.H & $\mathrm{H}$ & Mod & $\mathrm{L}$ & V.L \\
\hline $\begin{array}{l}\text { V.High } \\
(134)\end{array}$ & 134 & 0 & 0 & 0 & 0 \\
\hline High (635) & 0 & 635 & 0 & 0 & 0 \\
\hline $\begin{array}{l}\text { Moderate } \\
(257)\end{array}$ & 0 & 0 & 247 & 12 & 0 \\
\hline Low (135) & 0 & 0 & 0 & 135 & 0 \\
\hline V.Low(74) & 0 & 0 & 0 & 0 & 74 \\
\hline
\end{tabular}

Note: V.H means Very High, H means High, Mod means Moderate, L means Low and V.L Means Very Low.

$\mathrm{TP}=$ Class group correctly classified

$\mathrm{TN}=$ Class group incorrectly classified

$$
\begin{aligned}
\text { Detection Rate } & =\frac{T P}{T P+T N} \\
& =\frac{134+635+247+135+74}{134+635+247+135+74} \\
& =\frac{1225}{1225}=100 \%
\end{aligned}
$$

Table 2: Confusion Matrix of the Testing Set

\begin{tabular}{|l|l|l|l|l|l|}
\hline $\begin{array}{l}\text { Predicted as } \\
\text { Actual }\end{array}$ & V.H & H & Mod & L & V.L \\
\hline $\begin{array}{l}\text { V.High } \\
(134)\end{array}$ & 41 & 0 & 0 & 0 & 0 \\
\hline High (635) & 0 & 258 & 0 & 0 & 0 \\
\hline $\begin{array}{l}\text { Moderate } \\
(257)\end{array}$ & 0 & 0 & 42 & 0 & 0 \\
\hline Low (135) & 0 & 0 & 8 & 41 & 0 \\
\hline V.Low (74) & 0 & 0 & 0 & 0 & 18 \\
\hline
\end{tabular}

Note: V.H means Very High, H means High, Mod means Moderate, L means Low and V.L Means Very Low.

$\mathrm{TP}=$ Class group correctly classified

$\mathrm{TN}=$ Class group incorrectly classified

$$
\begin{aligned}
\text { Detection Rate } & =\frac{T P}{T P+T N} \\
& =\frac{41+258+42+41+18}{41+258+42+41+18+8} \\
& =\frac{400}{408}=98.04 \%
\end{aligned}
$$

Impressive results of $100 \%$ detection rate on the training set and $98.04 \%$ detection rate on the testing set were obtained.

\section{DEVELOPMENT OF A MOBILE APPLICATION FOR THE DIAGNOSIS OF MALARIA}

The mobile application was developed for the clinical diagnosis and treatment of malaria in android studio environment using PHP, HTML and Java as front end, MySQL as backend and Apache as the server. The rules generated by NNGE served as the engine room of the diagnosis while the medical experts provided the treatment in according to World Health Organization guidelines. The user-friendly mobile app has different modules in which users interact with. Some relational tables were created on MySQL to handle different data required for optimal functionality of the mobile application. There are four different modules associated with the mobile application as shown in Figure 1. 


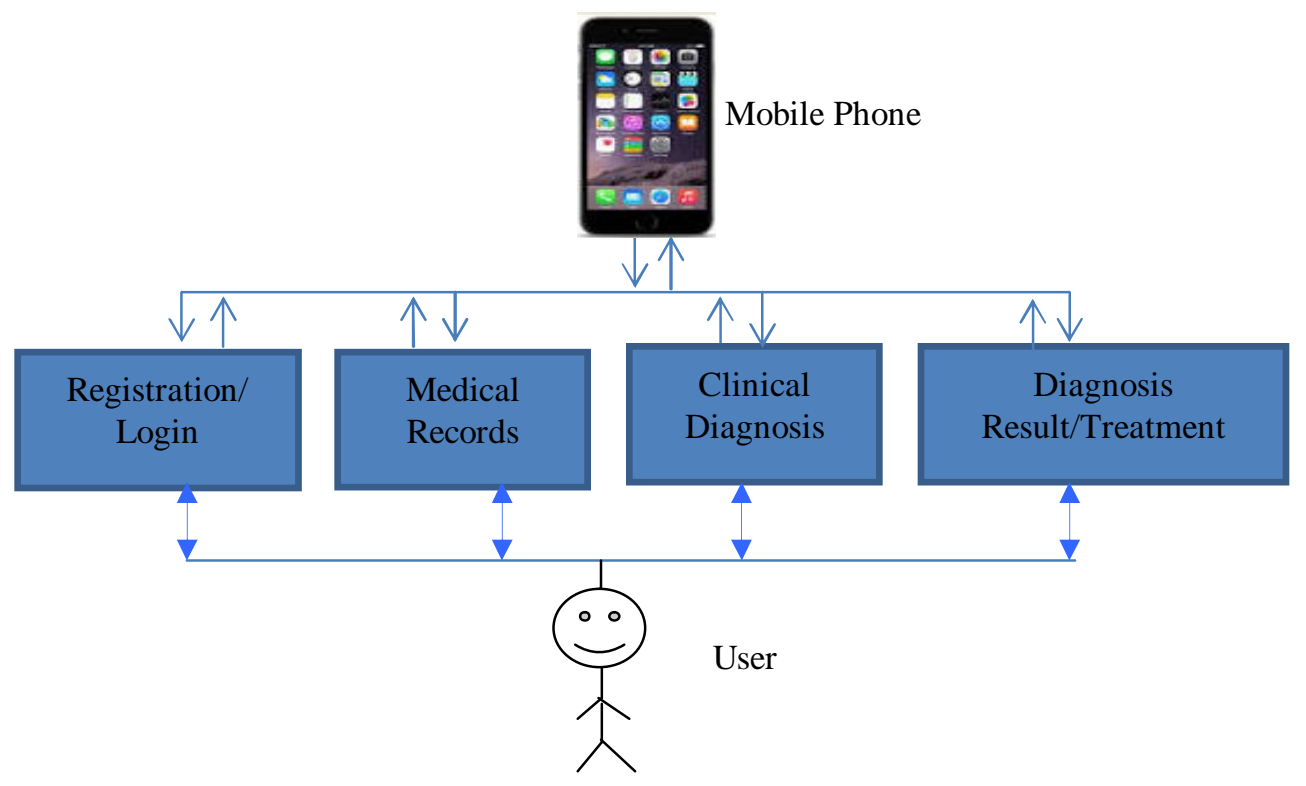

Figure 1: The mobile app and the associated modules

Registration and Login: This module handles the registration process of the new user. It collects the required information and stores it in the appropriate database on MySQL. This module is also empowered with mechanism to ensure authentication and login/access to the system.

Medical Record: Medical record module handles the medical history of the patients. Each patient has a unique portion of the database which handles his or her history with the system. Clinical Diagnosis: This module provides all the symptoms under consideration with options of severity to the user. The user interacts with it, make submission to the system and a clinical diagnosis is carried out.

Diagnosis Results and Treatment: The result of the diagnosis carried out in the diagnosis module is displayed in this module and the matching treatment already stored in one of the databases is provided. The screenshots of some of the mobile application interfaces are shown in Figure 2 to Figure 5. Figure 2 shows the login page, Figure 3 shows the registration page, Figure 4 displays user menu and Figure 5 shows the user self-test page.

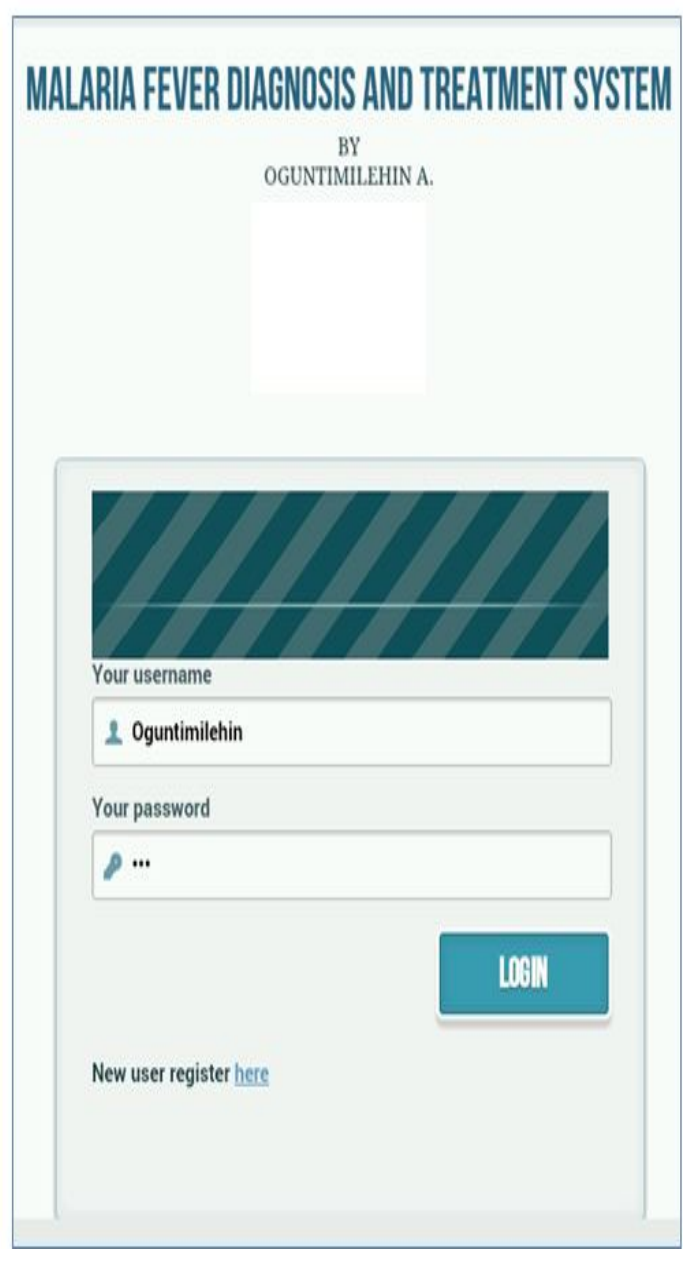

Figure 2: Login Page 


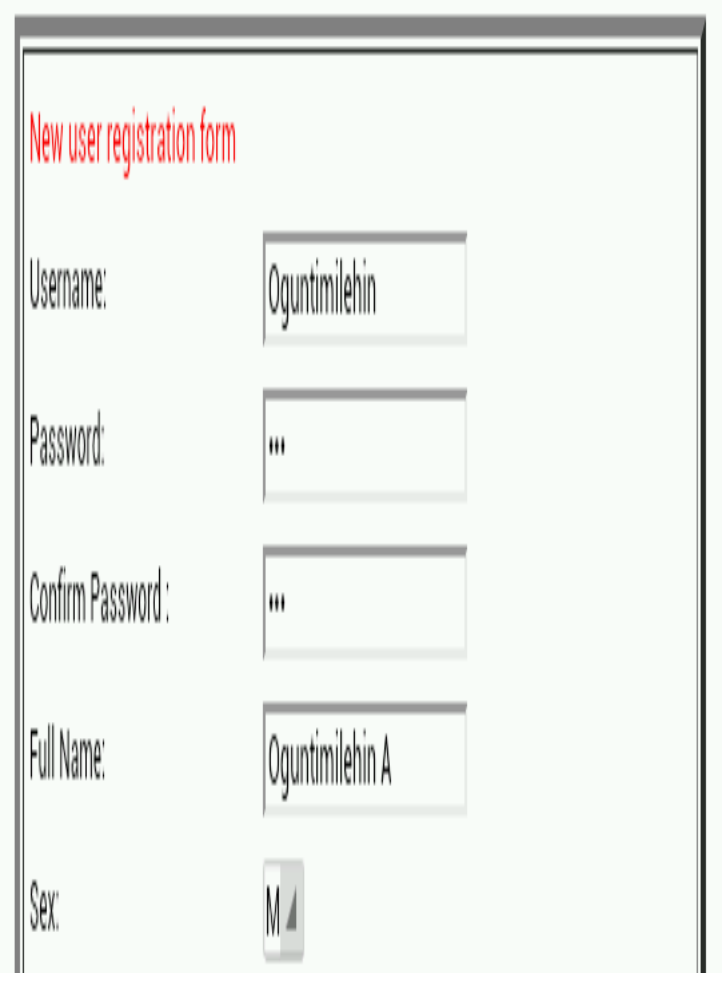

Figure 3: Registration Page

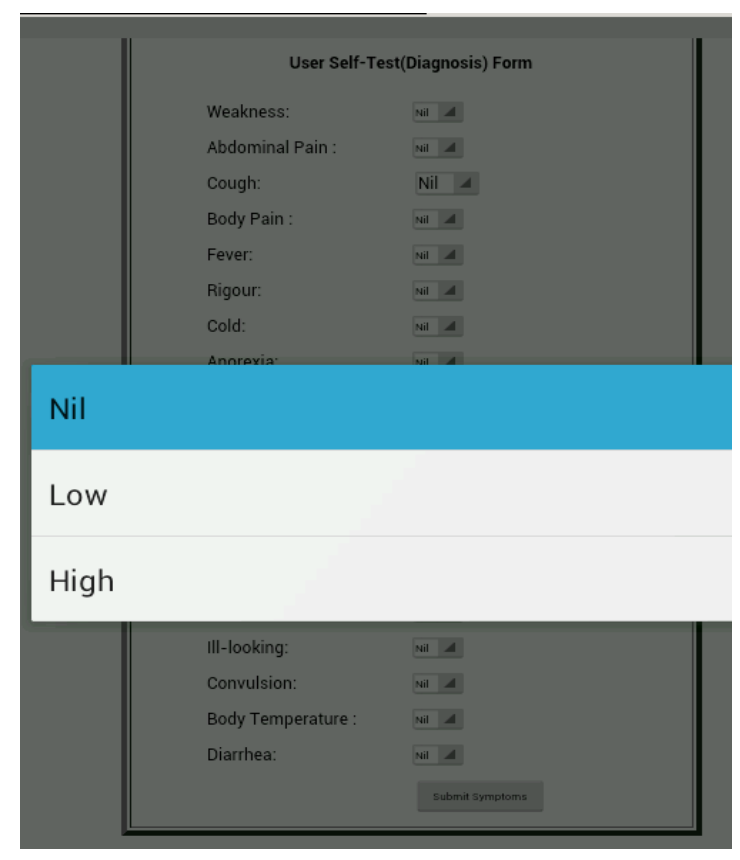

Figure 5: User Self-Test Page

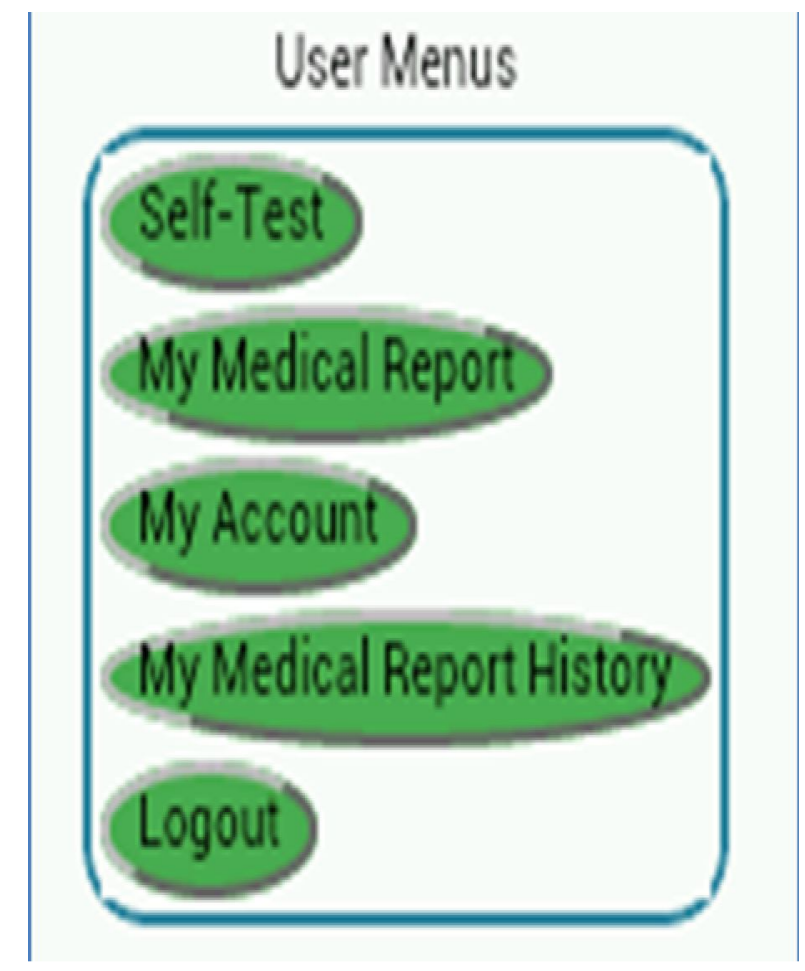

Figure 4: User menu or Dashboard

\section{CONCLUSION}

Considering the adverse effects of the dreaded malaria disease, solutions are worth looking for from every viable angle so as to reduce the number of deaths, spreading rate and overall burden. Provision of diagnosis system with improved accessibility by developing a mobile diagnosis application is desirable. A mobile application based malaria diagnosis system developed using a data mining technique-Non-Nested Generalized Exemplar (NNGE) as the engine room was developed in this work. The developed app will be of great advantage to humans, mostly in the malaria belt of Africa where the killer disease is highly prevalent.

\section{ACKNOWLEDGEMENT}

Sincere appreciation goes to the Chief Medical Director, medical staff and administrative staff of Adetoyin Hospital, Ado-Ekiti, Nigeria for their valuable inputs into this work.

\section{REFERENCES}

[1] Shahina B , A Case-Based Reasoning System for the diagnosis of individual sensitivity to stress in psychophysiology , Ph.D Thesis, School of Innovation, Design and Engineering, Malardelen University, 2009, Sweden. 
Oguntimilehin A., International Journal of Emerging Trends in Engineering Research, 8(2), February 2020, 259 - 264

[2] Nishad Nawaz, Artificial Intelligence Face Recognition for Applicant Tracking System, International Journal of Emerging Trends in Engineering Research, Volume 7, No. 12, pp. 8995-901, December, 2019.

[3] Djam X.Y, Wajiga G.M., Kimbi Y.H and Blamah N.V , A Fuzzy Expert System for the Management of Malaria, International Journal of Pure and Applied Sciences and Technology, Vol5, No2, Pg.84-108, 2011.

[4] Oguntimilehin A., Abiola O.B. and Odekunle K.A, Computer Aided Diagnostic Systems for Managing Typhoid Fever: A Review of Diagnosis Techniques, International Journal of Computer Applications, Vol 126(6), pp. 24-29, 2015. https://doi.org/10.5120/ijca2015906071

[5] V. Chandra Prakash, J.K.R. Satry B. Tirapathi Reddy, J.S. Ravi Teja, A. Bala Ven Katesh, M.S.K. Vamsi Varma, An Expert System for Building a Cognitive and Career Prediction Model Based on N-Coin Puzzle Game, International Journal of Emerging Trends in Engineering Research, volume 7, No. 11, pp. 410-416, November, 2019. https://doi.org/10.30534/ijeter/2019/037112019

[6] Obot O.U., Akinyokun O.C, and Udoh S.S, Application of Soft Computing Methodologies to the Management of Hypertension, Nigeria Computer Society $22^{\text {nd }}$ National Conference, Abeokuta, Nigeria, 2008, pp. 339-348.

[7] Agbonifo O.T. and Akinyede O.R., Modelling a Framework for Telemedicine in Transmitting Medical Data for Diagnosis Management in Nigeria, $22^{\text {nd }}$ National Conference Nigeria Computer Society, Abeokuta, Nigeria,2008, pp. 325- 332.

[8] Nana Y.A, mMES: A Mobile Medical Expert System for Health Institutions in Ghana, International Journal of Science and Technology, 2(6), pp. 333-343, 2012.

[9] Robert B., Larry C., Reza J, Thomas O. and Peter N, Leveraging Information Technology to Bridge the health workforce gap, World Health Organization, 2013, 91: pp. 890-892.

https://doi.org/10.2471/BLT.13.118737

[10] Oguntimilehin A., Adetunmbi A.O. and Abiola O.B , A Machine Learning Approach to Clinical Diagnosis of Typhoid Fever, International Journal of Computer and Information Technology, Vol 2(4), Pg. 671-676, 2013.

[11] NTG, Malaria, Centre for Disease Control, Northern Territory Government (NTG), 2012, www.nt.gov.au /health, Australia. Retrieved 04/05/18.

[12] Jiawei H. and Micheline K, Data Mining Concepts and Techniques ( $2^{\text {nd }}$ Edition), Morgan Kaufman Publishers (ELSEVIER), 2006, USA.

[13] Amal Found, Hossam M. Moftah Hesham A. MRI Brain Cancer Diagnosis Approach using Gabor Filter and Support Vector Machine, International Journal of Emerging Trends in Engineering Research, volume 7, No. 12, pp. 907-914, December, 2019. https://doi.org/10.30534/ijeter/2019/297122019

[14] Nils J.N, Introduction to Machine Learning, Amazon Press, 1998. http://www.scibd.com/doc/40480065 /Nils-J. Retrieved 26/02/2018.

[15] Adetunmbi A.O, Oguntimilehin A., and Falaki S.O, Web-Based Medical Assistant System for Malaria Diagnosis and Therapy, GESJ: Computer Science and Telecommunications, No 1(33), pp. 42-53, 2012.

[16] Prabhu S, Muhammed W, Asma A and Kathy A. J-T (2003), Decision Support Systems to Identify Different Species of Malari a Parasites, Annual Symposium Proceedings of American Medical Informatics Association (PMC1480132), 2003 USA.

[17] Vipul P, Pooja P. and Archana S, Computer Automation for Malaria Parasite Detection using Linear Programming, International Journal of Advanced Research in Electrical, Electronics and Instrumentation Engineering, Vol.2(5), pp.1984-1988, 2013.

[18] Priynka S., Singh D., Manoj K.B and Nidhi M , Decision Support System for Malaria and Dengue Disease Diagnosis (ASSMD), International Journal of Information and Communication Technology, Vol 3(7), pp. 633-640, 2013.

[19] Soomro A.A., Memon N.A, and Menopn M.S, Knowledge Based Expert System for Symptomatic Automated HealthCare, Sindh University Research Journal (Science Series), Vol 03 (I-A), pp. 79-84, 2011.

[20] Datta R.P. and Saha S (2011), An Empirical Comparison of Rule Based Classification Techniques in Medical databases, Working Paper, Indian Institute of Foreign Trade, Kolta Campus, 2011.

[21] Adem K., Dilek K. and Nizametin A, Benchmarking the Data Mining Algorithms with Adaptive Neuro-Fuzzy Inference System in GSM Churn Management, Source: Data Mining and Knowledge Discovery in Real Life Applications, Book edited by: Julio Ponce and Adem Karahoca, ISBN 978-3-902613-53-0, pp. 438, 2009 I-Tech, Vienna, Austria.

[22] Brent M, Instance Based Learning: Nearest Neighbour with Generalization, M.Sc Thesis, University of Waikato, New Zealand, 1995.

[23] Hall M., Frank E., Holmes G., Pfahringer B., Reutemann P., and Witten I., The WEKA Data Mining Software: An Update, SIGKDD Explorations, vol. 11, no. 1, 2009, pp. 10-18.

https://doi.org/10.1145/1656274.1656278 